\title{
The SKA view of cool-core clusters: evolution of radio mini-halos and AGN feedback
}

\author{
Myriam Gitti $^{* 1,2}$, Paolo Tozzi ${ }^{3}$, Gianfranco Brunetti ${ }^{2}$, Rossella Cassano ${ }^{2}$, Daniele \\ Dallacasa $^{1,2}$, Alastair Edge ${ }^{4}$, Stefano Ettori ${ }^{5}$, Luigina Feretti ${ }^{2}$, Chiara Ferrari $^{6}$, \\ Simona Giacintucci ${ }^{7,8}$, Gabriele Giovannini ${ }^{1,2}$, Michael Hogan ${ }^{4}$, Tiziana Venturi ${ }^{2}$ \\ 1 Dipartimento di Fisica e Astronomia - Università di Bologna, via Ranzani 1, I-40127 Bologna, \\ Italy; ${ }^{2}$ INAF - Istituto di Radioastronomia, via Gobetti 101, I-40129 Bologna, Italy; ${ }^{3}$ INAF - \\ Osservatorio Astrofisico di Arcetri, Largo E. Fermi 5, I-50125 Firenze, Italy; ${ }^{4}$ Department of \\ Physics, Durham University, Durham, DHI $3 L E$ UK; ${ }^{5}$ INAF - Osservatorio Astronomico di \\ Bologna, via Ranzani 1, I-40127 Bologna, Italy; ${ }^{6}$ Laboratoire Lagrange, UMR 7293, Université \\ de Nice Sophia-Antipolis, CNRS, Observatoire de la Côte d'Azur, 06300 Nice (FR); \\ ${ }^{7}$ Department of Astronomy, University of Maryland, College Park, MD 20742, USA; ${ }^{8}$ Joint \\ Space-Science Institute, University of Maryland, College Park, MD, 20742-2421, USA \\ E-mail: myriam.gitti at unibo.it
}

In about $70 \%$ of the population of relaxed, cool-core galaxy clusters, the brightest cluster galaxy (BCG) is radio loud, showing non-thermal radio jets and lobes ejected by the central active galactic nucleus (AGN). In recent years such relativistic plasma has been unambiguously shown to interact with the surrounding thermal intra-cluster medium (ICM) thanks to spectacular images where the lobe radio emission is observed to fill the cavities in the X-ray-emitting gas. This 'radio feedback' phenomenon is widespread and is critical to understand the physics of the inner regions of galaxy clusters and the properties of the central BCG. At the same time, mechanically-powerful AGN are likely to drive turbulence in the central ICM which may also play a role for the origin of non-thermal emission on cluster-scales. Diffuse non-thermal emission has been observed in a number of cool-core clusters in the form of a radio mini-halo surrounding the radio-loud BCG on scales comparable to that of the cooling region. Large mini-halo samples are necessary to establish their origin and connection with the cluster thermal properties and dynamics, especially in light of future X-ray characterization of the cluster cores as it is expected by Athena-XIFU. We show that All-Sky reference survey at Band 2 with SKA1 at confusion limit (rms $\sim 2 \mu \mathrm{Jy}$ per beam) has the potential to detect up to $\sim 620$ mini-halos at redshift $z<0.6$, whereas Deep Tier reference surveys at Band 1/2 with SKA1 at sub-arcsec resolution ( $\mathrm{rms} \sim 0.2 \mu \mathrm{Jy}$ per beam) will allow a complete census of the radio-loud BCGs at any redshift down to a $1.4 \mathrm{GHz}$ power of $10^{22} \mathrm{~W} \mathrm{~Hz}^{-1}$. We further anticipate that SKA2 might detect up to $\sim 1900$ new mini-halos at redshift $z<0.6$ and characterize the radio-mode AGN feedback in every cluster and group up to redshift $z \sim 1.7$ (the highest- $z$ where virialized clusters are currently detected) and even beyond, thus providing a complete picture of the feedback phenomenon in clusters and its role in shaping the large scale structure of the Universe.

Advancing Astrophysics with the Square Kilometre Array

June 8-13, 2014

Giardini Naxos, Sicily, Italy 


\section{Introduction}

The majority of baryons in galaxy clusters are in the form of diffuse hot plasma, the intracluster medium (ICM), heated mostly by gravitational processes at temperatures in the range 1-10 $\mathrm{keV}$ (going from groups to massive clusters) and visible in the X-ray band thanks to its thermal Bremsstrahlung emission. However the catastrophic cooling of the gas expected at the center of relaxed systems under these conditions (Fabian 1994) is not observed (e.g., Peterson \& Fabian 2006), suggesting that complex non-gravitational physical processes must be at work in the ICM to provide smoothly distributed heating on scales of about $100 \mathrm{kpc}$ and then stop the cooling process. Understanding the interplay of gravitational and non-gravitational physics in the ICM and its interaction with the relativistic plasma ejected by the central active galactic nucleus (AGN) is key for understanding the growth and evolution of galaxies and their central black holes, the history of star formation and the assembly of large-scale structures. In particular, the feedback from the central black hole has turned out to be an essential ingredient that must be taken into account in any model of galaxy formation and evolution. The main evidence of the action of radio-mode AGN feedback is in these 'cool-core' galaxy clusters, which hold a special place in the entire field of extragalactic astrophysics.

The central dominant $(\mathrm{cD})$ galaxies of cool-core clusters (which are characterized by short cooling times, high gas densities and low temperatures in the central regions) have a high incidence of radio activity, showing the presence of central FR-I radiogalaxies (Fanaroff \& Riley 1974) in $70 \%$ of the cases (Burns 1990; Dunn \& Fabian 2006; Best et al. 2007; Mittal et al. 2009). In particular, high-resolution X-ray observations performed with Chandra and XMM-Newton showed that the central radio sources have a fundamental and persistent effect on the ICM - the central hot gas in many cool-core systems is not smoothly distributed, but shows instead "holes" coincident with lobes of extended radio emission. The most typical configuration is for jets from the central dominant elliptical of a cluster to extend outwards in a bipolar flow, inflating lobes of radio-emitting plasma (radio 'bubbles') which are spatially separated from the thermal ICM component. The interpretation is that these lobes push aside the X-ray-emitting gas of the cluster atmosphere, thus excavating 'cavities' in the ICM which are detectable as deficits in the X-ray images. Thanks to spectacular images where the radio emission is observed to fill the cavities in the X-ray emitting ICM, the radio galaxies have thus been identified as a primary source of feedback to solve the so-called 'cooling flow problem' (for recent reviews see Gitti et al. 2012; McNamara \& Nulsen 2012; Fabian 2012). In Figure 1 we show clear examples of radio lobes (black contours) filling the cavities in the ICM in a galaxy group (left panel) and in a massive cluster (right panel).

The detailed mechanism to transfer the mechanical energy of the radio jets into thermal energy of the ICM is still unclear. It has been proposed that mechanically-powerful AGN are responsible for driving turbulence in the central ICM, which must eventually dissipate into heat thus contributing to offsetting radiative cooling (e.g., Zhuravleva et al. 2014). On the other hand, such a turbulence may also contribute to re-accelerating a seed population of (sub-)relativistic particles naturally present in the ICM, and producing diffuse non-thermal emission. Obvious sources of the seed relativistic electrons are, for example, the buoyant radio bubbles that are inflated by the radio activity of the central AGN itself and disrupted by gas motions in the core.

\footnotetext{
* Speaker.
} 

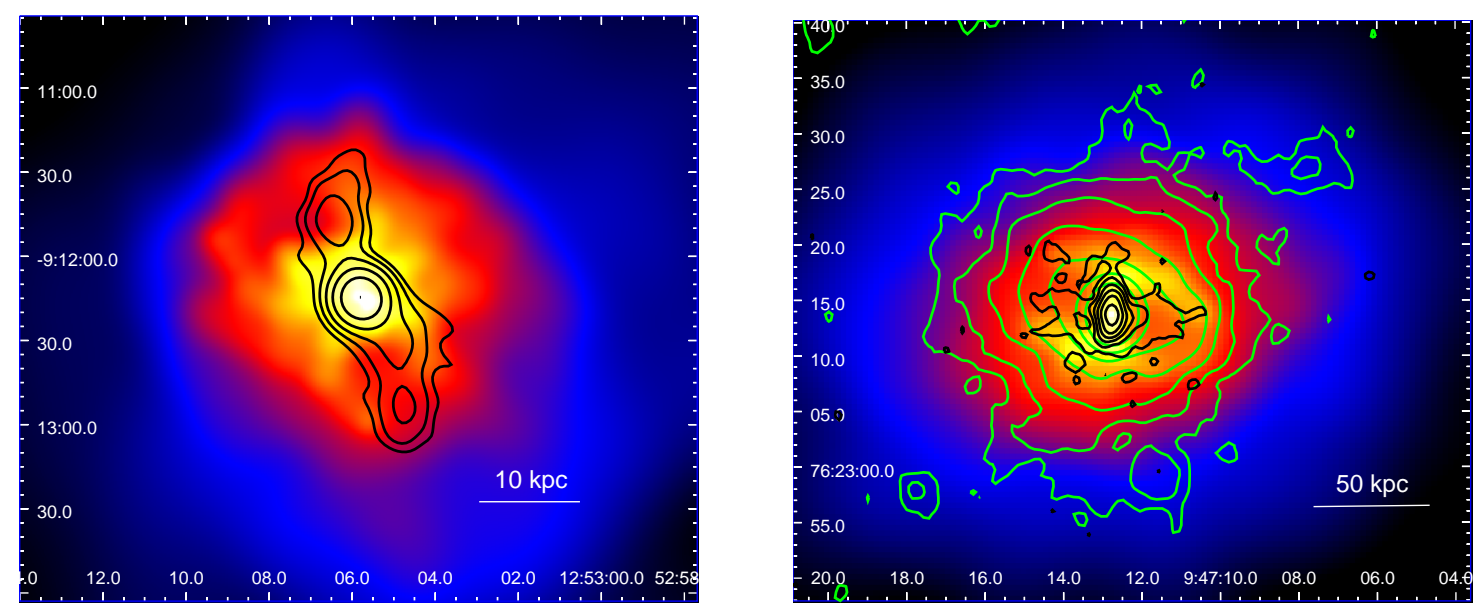

Figure 1: Left panel: X-ray image of the galaxy group HCG 62 at $z=0.0137$ (Gitti et al. 2010) with superimposed GMRT radio contours at $610 \mathrm{MHz}$. Right panel: X-ray image of the galaxy cluster RBS 797 at $z=0.35$ with superimposed VLA radio contours at $1.4 \mathrm{GHz}$ (green contours, Gitti et al. 2012; Doria et al. 2012) and at $5 \mathrm{GHz}$ (black contours, Gitti et al. 2013). In both cases X-ray cavities in the ICM are observed to be coincident with the BCG radio lobes (black contours), and in the case of RBS 797 a large-scale radio mini-halo is also detected (green contours).

Diffuse non-thermal emission has been observed in a number of cool-core clusters, where the radio-loud brightest cluster galaxy (BCG) is surrounded by a so-called 'radio mini-halo'. Minihalos are diffuse, faint radio sources observed at the cluster center on scales (total size) 100-500 kpc comparable to that of the cooling region, with steep radio spectra and amorphous (roundish) shape. The typical mini-halo power at $1.4 \mathrm{GHz}$ is of the order of $\sim 10^{23}-10^{24} \mathrm{~W} \mathrm{~Hz}^{-1}$. These radio sources are not directly connected with the radio bubbles, but the mini-halo emission is on larger scales and is truly generated from the ICM where the thermal plasma and the relativistic electron population are mixed (see in the right panel of Figure 1 the example of the cluster RBS 797 which hosts both a mini-halo, in green contours, and a cavity-bubble system, in black contours). Therefore, the synchrotron emission from radio mini-halos proves that $\sim \mathrm{GeV}$ electrons are diffusing through $\sim \mu \mathrm{G}$ magnetic fields permeating the ICM, as in the case of giant radio halos and relics (see also Cassano et al. 2015; Ferrari et al. 2015; Govoni et al. 2015). Mini-halos are qualitatively similar to the most extreme case of giant radio halos and relics that however are typically found in disturbed/non cool-core clusters and are most likely powered by cluster mergers. In fact, radio mini-halos indicate that, in addition to mergers, diffuse non-thermal emission can be powered also by less extreme processes that dissipate energy associated to large-scale (tens-hundreds kpc) motions at micro-physical scales and are connected at some level with the mechanisms responsible for the heating of the cooling gas. Although mini-halos are not directly sustained by the central engine, it is nonetheless likely that the AGN plays a role for the origin of these sources. For example, it has been proposed that the relativistic electrons that generate mini-halos are released in the ICM by the central AGN and then can be transported and re-accelerated by turbulent motions in the cool cores (Gitti et al. 2002; Mazzotta \& Giacintucci 2008; ZuHone et al. 2013).

Only about twenty mini-halos (or candidates) are known so far, all at redshift $z<0.6$ (Giacin- 
tucci et al. 2014b; van Weeren et al. 2014). Due to the limitations of current radio interferometers it is difficult to assess the occurrence of radio mini-halos in clusters. In particular, the mini-halo detection is complicated by the need of separating their diffuse, low surface brightness emission (at a level of few $\mu \mathrm{Jy} \operatorname{arcsec}^{-2}$ at $1.4 \mathrm{GHz}$ ) from the bright emission of the central radio BCG. This requires a very good sensitivity to diffuse emission, high dynamic range and good spatial resolution, which will be achievable with SKA only. A large mini-halo sample observed by SKA will allow us to reach a better understanding of this class of sources, in particular to establish their origin and connection with the cluster thermal properties and dynamics which will be characterized at unprecedented levels by the next-generation of X-ray instruments like XIFU, the X-ray Integral Field Unit covering the 0.2 to $10 \mathrm{keV}$ energy range with unrivalled energy resolution, that will flight onboard of the ESA-L2 mission Athena ${ }^{1}$ (expected launch in 2028). Furthermore, at present the study of the radio-mode feedback in clusters is limited to $z<0.7$ with very few cases at high redshift (Hlavacek-Larrondo et al. 2012), while virialized clusters are currently detected up to redshifts of 1.6 or larger (at present the highest redshift, massive cluster whose virialization is confirmed by deep X-ray study is XDCP J0044.0-2033 at $z=1.58$, Tozzi et al., submitted). Therefore, there is a wide redshift range where radio-mode feedback is still unexplored. In addition, recent studies show that cool cores are already present and well developed at least at $z \sim 1$, suggesting that cool-core formation takes place on a short time-scale after the formation of the cluster (Santos et al. 2010, 2012). Since the association of cool cores with radio mini-halos and radio AGN is ubiquitous (Feretti et al. 2012; Sun 2009), with SKA we expect to be able to trace the non-thermal emission and feedback activity in clusters, and therefore to study the interplay between thermal and non-thermal plasma in the central regions of galaxy clusters, up to the highest redshift where clusters are found.

The non-thermal activity and radio-mode feedback in clusters of galaxies is a scientific case key to the physics of the ICM and the evolution of the cosmic large scale structures. Radio astronomy in the SKA era can be the main scientific driver in these fields.

In the rest of this chapter we assume a $\Lambda \mathrm{CDM}$ cosmology with $H_{0}=70 \mathrm{~km} \mathrm{~s}^{-1} \mathrm{Mpc}^{-1}$ and $\Omega_{M}=1-\Omega_{\Lambda}=0.3$.

\section{Origin of radio mini-halos}

At a first glance, radio mini-halos may resemble a small-scale version of giant radio halos, extending on scales of the order of few hundred kpc size rather than Mpc size. However, the two classes of radio sources show prominent differences. First of all, in clear contrast to giant radio halos ${ }^{2}$, mini-halos are always found in dynamically relaxed systems suggesting that cluster mergers do not play a major role for their origin. Also the synchrotron volume emissivity of mini-halos is typically larger than that of giant halos (Cassano et al. 2008; Murgia et al. 2009). On the other hand, it is yet not well established whether the underlying physical mechanisms that accelerate the cosmic ray electrons (CRe) in mini-halos differ substantially from the analogous mechanisms in giant radio halos. Clusters hosting mini-halos always have a central radio-loud AGN, which often exhibits outflows in the form of radio lobes and bubbles injecting CRe into the central regions of

\footnotetext{
${ }^{1}$ http://www.the-athena-x-ray-observatory.eu/

${ }^{2}$ note that there are also two atypical cases of giant halos, that apparently are in clusters without strong merging activity (Farnsworth et al. 2013; Bonafede et al. 2014)
} 
galaxy clusters. In principle these AGN could represent the primary source of the CRs in minihalos, however they are not sufficient by themselves, at least without some dynamical contribution, to explain the diffuse radio emission. In particular, a slow diffusion problem exists for mini-halos (as for giant halos); that is, the energy loss time scale of the radio emitting $\mathrm{CRe}\left(\approx 10^{8} \mathrm{yr}\right)$ is much shorter than the time needed by these particles to diffuse efficiently across the emitting volume (e.g., Gitti et al. 2004). Similarly to giant halos, two physical mechanisms have been identified as possibly responsible for the radio emission in mini-halos: i) re-acceleration of CRe (leptonic models or re-acceleration models) where electrons are reaccelerated by micro-turbulence in the radio-emitting region, and ii) generation of secondary CRe (hadronic or secondary models) where the radio-emitting region is continuously supplied with secondary electrons generated by inelastic collisions between cosmic-ray protons (CRp) and thermal protons (see Brunetti \& Jones 2014, for a review).

A key question in the re-acceleration models is the origin of the turbulence responsible for reaccelerating the electrons. Gitti et al. (2002) originally considered magnetohydrodynamic (MHD) turbulence amplified by compression in the cool cores, where the necessary energetics to power radio mini-halos is supplied by the cooling flow process itself. This model predicts a direct connection between the synchrotron emitted power in mini-halos and the energetics of (or energy available into) the cooling flow. Remarkably a trend between the radio power and the cooling flow power is observed in mini-halo clusters (Gitti et al. 2004, 2007, 2012), where the strongest radio mini-halos are associated with the most powerful cooling flows, thus hinting at a direct connection between the thermal properties of cooling flows and the non-thermal emission of radio mini-halos. Although these observations support a connection between synchrotron and (gas-)cooling power, the physical mechanisms responsible for the acceleration of the emitting electrons are still poorly constrained. In particular, in the re-acceleration model the origin of the turbulence necessary to trigger the electron re-acceleration is still debated. As already mentioned in the Introduction, one possibility is that the turbulence is powered by AGN activity. In this case the mechanisms responsible for particles re-acceleration in mini-halos and for the gas heating should be intimately connected. This scenario can be tested by investigating whether all mini-halo clusters show evidence of AGN feedback (see Sect. 4). On the other hand, the signatures of minor dynamical activity have recently been detected in some mini-halo clusters, thus suggesting that additional or alternative turbulent energy may be provided by minor mergers (Gitti et al. 2007; Cassano et al. 2008) and related gas sloshing mechanism in cool-core clusters (Mazzotta \& Giacintucci 2008; Giacintucci et al. 2014a), which is also supported by recent simulations (ZuHone et al. 2013). The clearest observational signatures of these large-scale gas motions are spiral-shaped cold fronts seen in the majority of cool-core clusters (Markevitch \& Vikhlinin 2007). These cold fronts are believed to be produced by the cold gas of the core sloshing in the clusters deep potential well, in response to crossing dark matter subhalo motions, for example. Those sloshing motions can advect ICM across the cluster core where turbulence can also be produced (Fujita et al. 2004; Ascasibar \& Markevitch 2006; ZuHone et al. 2010).

Understanding the nature of mini-halos would provide invaluable information on the microphysics of the ICM, including the process of amplification and dissipation of the magnetic field, and on the diffusion/transport of CRs in these environments. Both understanding the nature of turbulence in cool-core clusters and discriminating between a leptonic and hadronic origin of radio 
mini-halos is however very challenging due to severe limitations of current observational constraints. A leap forward is expected from future spectral and polarimetric information in the radio band, that will become available with SKA, combined with constraints in other bands (e.g., hard $\mathrm{X}$-rays and gamma-rays). For example, the combination of SKA1-LOW and SKA1-MID/SUR observations will allow us to establish whether the spectrum of mini-halos is curved or if it is a pure power-law. Compelling evidence for very steep spectra and/or for the existence of spectral breaks at high frequencies would favour a re-acceleration scenario rather than a hadronic origin of the mini halos (Brunetti \& Jones 2014). At the same time, polarimetric information will allow us to probe the turbulent status of the magnetic field in the radio-emitting region, whereas the degree of circular polarization will allow us to poinpoint the $\mathrm{CRe}$ to $\mathrm{CRp}$ ratio, thus constraining the hadronic or re-acceleration origin of radio mini-halos.

In this chapter we focus on the crucial information that will be obtained by statistical studies with SKA. Although a correspondence between radio mini-halos and cool-core clusters is well established, current radio studies do not provide an exhaustive view of the occurrence of radio mini-halos in galaxy clusters more generally. For example, it is still not clear whether these radio sources are common or rare in cool cores. Statistical studies of large cluster samples are necessary to reach a better understanding of these sources, investigating in particular the following key points:

- Do all cool-core clusters host a radio mini-halo? How does the mini-halo/cool-core fraction evolve with redshift? How do the radio properties correlate with the X-ray properties?

(radio power-limited sample with wider redshift distribution, synergy with eROSITA and Athena $X$-ray satellites)

- Does the central AGN play a significant role in powering radio mini-halos? What is the fraction of mini-halo clusters that show evidence of AGN feedback?

(spectral studies, radio bubbles filling the $X$-ray cavities)

- Are mini-halos intrinsically different from giant halos, or do they represent a different evolutionary stage of the same non-thermal phenomenon? In particular, if non cool-core clusters evolve into cool-core clusters as believed, do giant radio halos evolve into mini-halos?

(polarimetric studies, evolutive models and synergy with Athena $X$-ray satellite)

We will present evidence that surveys with SKA1 and eventually with SKA2 will have the capabilities to address the above questions.

\section{Statistics of radio mini-halos}

We start from the list of mini-halos reported in Giacintucci et al. (2014b) who recently selected a large sample of X-ray-luminous clusters with available high-quality radio data, and discovered four new mini-halos. By excluding the objects that they classify as "candidate" or "uncertain", and by including the new detection in the Phoenix cluster (van Weeren et al. 2014), the mini-halo sample used in this work comprises the following 16 objects (sorted by decreasing radio power at 1.4 GHz): RX J1347.5-1145 ( $\mathrm{z}=0.451)$, Phoenix ( $\mathrm{z}=0.596)$, RX J1720.1+2638 ( $\mathrm{z}=0.159)$, A 2390 (z=0.23), RX J1532.9+3021 ( $\mathrm{z}=0.362)$, RXC J1504.1-0248 ( $\mathrm{z}=0.215)$, RBS 797 ( $\mathrm{z}=0.35)$, Perseus ( $\mathrm{z}=0.018)$, MS 1455.0+2232 ( $\mathrm{z}=0.258), \mathrm{ZwCl} 3146$ ( $\mathrm{z}=0.290), \mathrm{A} 1835$ ( $\mathrm{z}=0.252)$, A 2204 ( $\mathrm{z}=0.152)$, A $478(\mathrm{z}=0.088)$, A 2029 ( $\mathrm{z}=0.077)$, Ophiuchus ( $\mathrm{z}=0.028), 2$ A $0335+096(\mathrm{z}=0.035)$. 

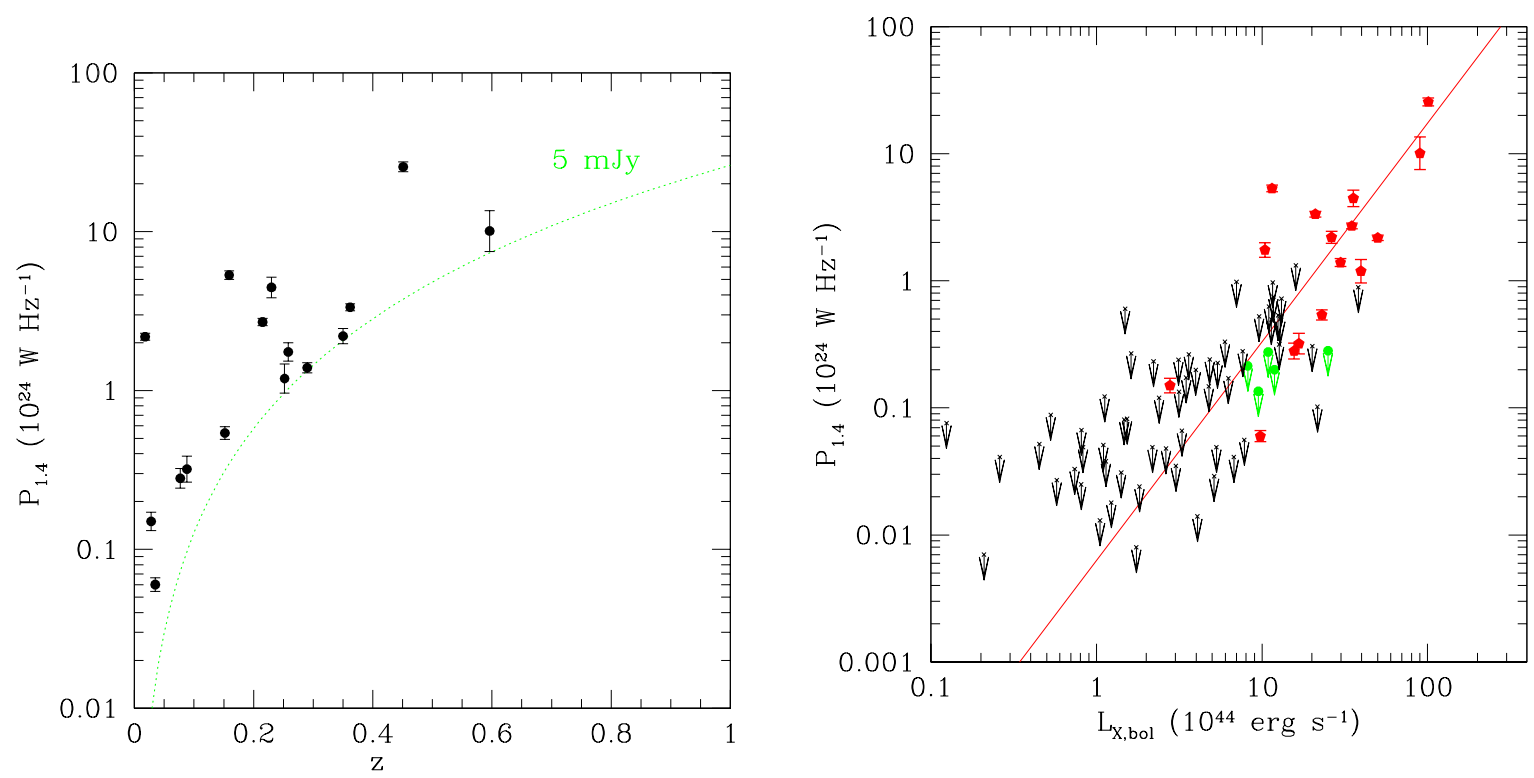

Figure 2: Left panel: Radio power at $1.4 \mathrm{GHz}$ versus redshift for the mini-halo sample. The green dotted line represents the radio power corresponding to a flux density of $5 \mathrm{mJy}$. Right panel: Radio power at 1.4 $\mathrm{GHz}$ versus the cool-core-excised bolometric X-ray luminosity for the mini-halo sample (red filled circles). The red solid line is the best fit relation to the red points from bisector BCES regression to the parameters in log space (see Eq. 3.1). The green points are the observed upper limits from Kale et al. (2013), whereas the black crosses are the current upper limits estimated for the ACCEPT clusters which are candidates to host radio mini-halos.

We note that all these mini-halo clusters have a central entropy $K_{0}=k T_{0} n_{0}^{-2 / 3} \lesssim 25 \mathrm{keV} \mathrm{cm}^{2}$ (where $K 0$ values are taken from the Chandra ACCEPT ${ }^{3}$ sample of Cavagnolo et al. 2009), that according to the classification of Hudson et al. (2010) defines the population of strong cool cores (SCC). Recently, Panagoulia et al. (2014) take issues with the ACCEPT sample, arguing that the flattening of the entropy profile towards smaller radii, that is, the measured excess 'floor' $K_{0}$ of the core entropy above the best-fitting power-law profile for the entropy at larger radii, could be a resolution effect. Since the measured central entropy is correlated with the central bin size, this problem is enhanced for clusters with poor data quality, or poor spatial resolution, where the inner regions are undersampled. However, we note that this effect is not an issue in this context, as it simply implies that the true central entropy value could be even lower, thus strengthening the SCC classification of our mini-halo sample (see also Giacintucci et al. in preparation for a more detailed investigation).

In Figure 2 (left panel) we plot the $1.4 \mathrm{GHz}$ radio power of the mini-halos (taken from Giacintucci et al. 2014b; van Weeren et al. 2014) versus redshift. For comparison we overlay the

\footnotetext{
${ }^{3}$ Archive of Chandra Cluster Entropy Profile Tables
} 
radio power corresponding to a flux of $5 \mathrm{mJy}$ (green dotted line), which is roughly the lowest mini-halo flux detected up to now. It is evident that there is a strong observational bias that limits our present ability of detecting mini-halos; that is, we are currently missing many faint mini-halos simply because of the limited sensitivity of present radio telescopes. But how many mini-halos await discovery? The technical improvement reachable with SKA will be fundamental to answer to this question. To estimate the quantum leap produced by SKA in the ability of detecting radio mini-halos, we must link the non-thermal properties of these sources with the thermal properties of their host galaxy clusters. Being dominated by selection effects, the current radio information on mini-halos does not allow us to make predictions on future discoveries. By contrast, cluster statistics in terms of X-ray properties is already available from Chandra and XMM studies, and can be exploited to forecast future detections of radio mini-halos, provided an intrinsic relation between the thermal and non-thermal cluster properties exists.

To link the properties of the mini-halos with the global X-ray properties of the host clusters, in Figure 2 (right panel) we plot with red filled circles the mini-halo radio power at $1.4 \mathrm{GHz}$ versus the cool-core-excised bolometric X-ray luminosity of the cluster, taken from the ACCEPT sample. We used the bivariate correlated error and intrinsic scatter (BCES) algorithm (Akritas \& Bershady 1996) to perform regression fits to the data in log space, determining the best-fitting powerlaw relationship (bisector method) between the radio power, $P_{1.4}$, and the bolometric X-ray luminosity, $L_{\mathrm{X}, \text { bol }}$ (overlaid as a red solid line in Figure 2, right panel):

$$
\log P_{1.4}=1.72( \pm 0.28) \log L_{\mathrm{X}, \mathrm{bol}}-2.20( \pm 0.46)
$$

where $P_{1.4}$ is in units of $10^{24} \mathrm{~W} \mathrm{~Hz}^{-1}$ and $L_{\mathrm{X}, \mathrm{bol}}$ is in units of $10^{44} \mathrm{erg} \mathrm{s}^{-1}$. We note that our analysis improve significantly the previous ones (e.g., Cassano et al. 2008; Kale et al. 2013) thanks to the better statistics, and that a similar scaling (with slope $1.76 \pm 0.16$ ) is obtained for giant radio halos (Brunetti et al. 2009). It is also entirely plausible that the flux density of mini-halos scales, perhaps even better, with other X-ray properties as well, such the cool-core strength ${ }^{4}$. However, we stress that for statistical studies of large cluster samples it is essential to link the non-thermal properties of the mini-halos to the thermal properties of the ICM which are easily observable with $\mathrm{X}$-ray surveys, such as the X-ray luminosity.

Our starting point is the optimistic zero-th order assumption that every SCC cluster hosts a radio mini-halo, and that such a mini-halo follows the radio-X-ray power correlation. To check whether our hypothesis is consistent with the current independent constraints, in Figure 2 (right panel) we report in green the observed upper limits to the mini-halo emission in 5 SCC clusters from Kale et al. (2013). Although these limits appear systematically lower than the correlation, they are still consistent with the scatter of the observed trend. In Figure 2 (right panel) we also report the radio upper limit distribution of all SCC clusters in the ACCEPT sample which are candidate to host radio mini-halos; that is, according to our assumption, clusters having a central entropy $K 0 \lesssim 25 \mathrm{keV} \mathrm{cm}^{2}$. Although most of these clusters have radio observations, there is no evidence for the presence of mini-halos in these clusters (see Giacintucci et al. 2014b). Thus a mandatory point is to check whether the absence of mini-halos in these SCC clusters is still consistent with

\footnotetext{
${ }^{4}$ a detailed investigation of this correlation, which requires accurate model fits to the X-ray spectra extracted inside the cooling region, is currently underway and will be presented in a forthcoming paper
} 
our assumptions. We did that by estimating the radio upper limits in Figure 2 (right panel) based on the capabilities of current radio telescopes. In particular we adapted to mini-halos the criterion of Cassano et al. (2012) based on a threshold in flux for giant halos. From this threshold we derive the minimum flux of radio mini-halos that can be detected by assuming a spatial distribution of their brightness, and use this value to derive the upper limit to the luminosity of mini-halos in each SCC cluster. Murgia et al. (2009) modeled the radio brightness profile $I(r)$ of radio mini-halos with an exponential of the form $I(r)=I_{0} \exp \left(-r / r_{e}\right)$, where $r_{e}$ is the effective radius. This implies that the outermost, low brightness, regions of mini-halos are very difficult to detect. However, what is important is the capability to detect at least the brightest mini-halo regions. By considering the brightness profile of Murgia et al. (2009), we estimate that radio mini-halos emit about half of their total radio flux within their half radius $r_{50} \sim 1.68 r_{e}$. Following Cassano et al. (2015) we can derive the minimum detectable flux, $f_{\min }(z)$, by assuming that the mini-halo is detected when the integrated flux within $r_{50}$ gives a signal to noise ratio $\xi_{2}$. In particular, for each low-entropy cluster selected in the ACCEPT sample, we estimate $f_{\min }(z)$ from Eq. 4.2 of Cassano et al. (2015) by considering the angular size of the radio mini-halo in arcseconds, $\theta_{\mathrm{MH}}(z)$, which corresponds to $2 r_{50}$ at the given cluster redshift. We adopt typical values of the current sample of observed minihalos (e.g., Giacintucci et al. 2014b) of half radius $r_{50} \sim 100 \mathrm{kpc}$, rms noise per beam $\mathrm{F}_{\text {rms }}=25$ $\mu \mathrm{Jy}, \xi_{2}=10$, beam angular size $\theta_{b}=10$ arcsec. The observed (green points) and estimated (black crosses) upper limits in Figure 2 (right panel) do not violate the $P_{1.4}-L_{\mathrm{X}, \text { bol }}$ correlation, therefore we shall proceed with the assumption that all SCC clusters host a mini-halo that follows the radio$\mathrm{X}$-ray power correlation.

To derive a zeroth-order estimate of the detection limit reachable by SKA, in Figure 3 (left panel) we plot the clusters in the ACCEPT sample which are candidates to host radio mini-halos

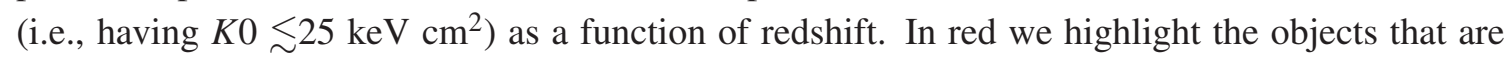
known to possess a mini-halo, and in blue overlay the threshold in X-ray bolometric luminosity obtained by converting the upper limits on $P_{1.4}$ (black points in Figure 2, right panel) to upper limits on $L_{X}$,bol by means of Eq. 3.1. The blue line thus is representative of the current mini-halo detection limit on the population of SCC clusters. As clear, at present we are able to investigate only the "tip of the iceberg" of the population of SCC clusters. We then estimate the minimum flux as described above by assuming values in the reach of SKA1-SUR surveys at confusion limit (see Prandoni \& Seymour 2015; Cassano et al. 2015, for more discussion on the role of confusion). In particular, the red solid line in Figure 3 (left panel) represents the minimum X-ray bolometric luminosity obtained (via the $P_{1.4}-L_{\mathrm{X}, \text { bol }}$ correlation given by Eq. 3.1) from the minimum $1.4 \mathrm{GHz}$ radio power calculated for observations at $1.4 \mathrm{GHz}$ with $\mathrm{F}_{\mathrm{rms}}=2 \mu \mathrm{Jy}, \xi_{2}=10, \theta_{b}=8$ arcsec. We also estimate that the scientific outcomes delivered in this field during early science operations of SKA1 (with performances at $\sim 50 \%$ ) will be significant, allowing the radio follow-up to more than $70 \%$ of the ACCEPT sample (see red dotted line in Figure 3, left panel). Our anticipations for SKA2 are shown in Figure 3, left panel (red dashed line).

\subsection{Number of radio mini-halos expected in surveys with SKA1-SUR}

Given the large number of stations and the unprecedented $(u, v)$ coverage of SKA, the detection will depend only on the mini-halo flux density. The number of mini-halos that can be detected from a radio survey with a given sensitivity up to a certain redshift $z$ is computed by integrating the radio 

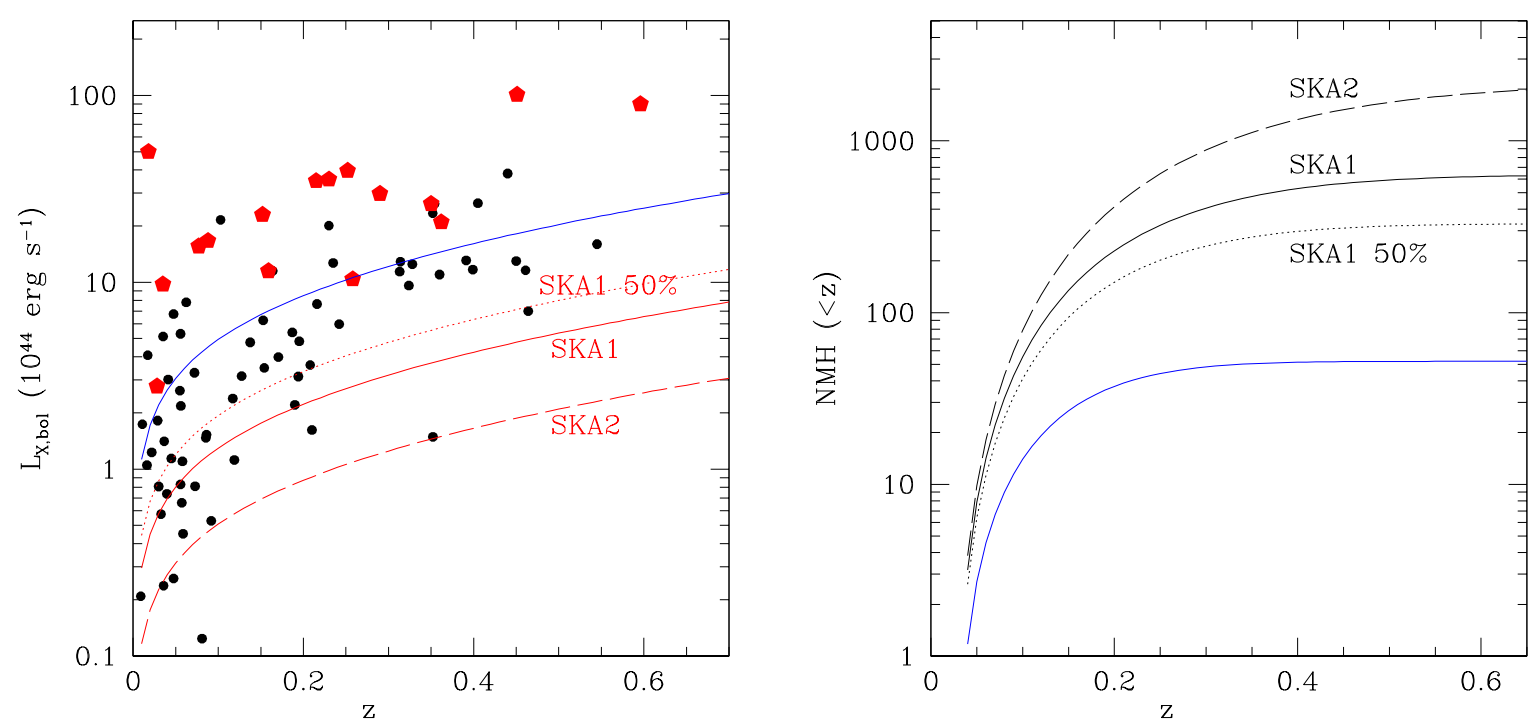

Figure 3: Left panel: Cool-core-excised bolometric X-ray luminosity, $L_{\mathrm{X} . \text { bol }}$, versus redshift for the ACCEPT clusters with central entropy $K 0 \lesssim 25 \mathrm{keV} \mathrm{cm}^{2}$ (Cavagnolo et al. 2009). The clusters hosting a radio mini-halo are highlighted in red. The blue solid line is indicative of the current mini-halo detection limit on the population of SCC clusters $\left(\mathrm{F}_{\mathrm{rms}}=25 \mu \mathrm{Jy}, \theta_{b}=10 \operatorname{arcsec}\right)$, whereas the red solid line represents the detection limit reachable by SKA1-SUR surveys at confusion limit (obtained from the minimum radio power calculated for observations at $1.4 \mathrm{GHz}$ with $\mathrm{F}_{\mathrm{rms}}=2 \mu \mathrm{Jy}, \theta_{b}=8 \mathrm{arcsec}$ ). The red dotted lines and red dashed lines represent the detection limit reachable by early science operations of SKA1-SUR $\left(\mathrm{F}_{\mathrm{rms}}=4 \mu \mathrm{Jy}, \theta_{b}=8\right.$ arcsec), and by SKA2 ( $\mathrm{rms}=0.2 \mu \mathrm{Jy}, \theta_{b}=4 \mathrm{arcsec}$ ), respectively. Right panel: Integrated number of radio mini-halos detectable at $1.4 \mathrm{GHz}$ out to $z, \mathrm{NMH}(<z)$, as a function of redshift. The predictions for All Sky surveys with SKA1, early SKA1 and SKA2 are shown with black solid lines, black dotted lines and black dashed lines, respectively. The prediction for a hypothetical survey conducted with current telescopes is also shown for comparison (blue solid line). The survey performances adopted to estimate the integrated number of mini-halos are the same as in left panel. The uncertainty envelopes driven by the $P_{1.4}-L_{\mathrm{X}}$ correlation alone are of the order of $\sim 30 \%$ at $\mathrm{z}=0.1, \sim 40 \%$ at $\mathrm{z}=0.3$ and $\sim 50 \%$ at $\mathrm{z}=0.6$, but are not shown here to avoid confusion among different lines.

luminosity function (RLF) of mini-halos $\frac{d N_{\mathrm{MH}}}{d P_{1.4} d V}$ over radio luminosity and redshift (cf. Eq. 5.1 of Cassano et al. 2015), where the minimum radio luminosity detectable at a given redshift can be estimated from $f_{\min }(z)$ as described above (Eq. 4.2 of Cassano et al. 2015). Under our assumptions the RLF of mini-halos (per sky area surveyed in steradians) is

$$
\frac{d N_{\mathrm{MH}}}{d P d V}=\mathrm{f}_{\mathrm{SCC}} \frac{d N_{\mathrm{cl}}}{d L_{\mathrm{X}} d V} \frac{d L_{\mathrm{X}}}{d P_{1.4}}
$$

where $\frac{d N_{\mathrm{cl}}}{d L_{\mathrm{X}} d V}$ is the X-ray luminosity function (XLF) of galaxy clusters (e.g., Mullis et al. 2004; 
Böhringer et al. 2014), $\mathrm{f}_{\mathrm{SCC}}$ is the fraction of clusters with SCC (e.g., Hudson et al. 2010; Bharadwaj et al. 2014) and $\frac{d L_{X}}{d P_{1.4}}$ can be obtained from the observed radio-X-ray power correlation for mini-halos.

In particular, here we adopt the evolving XLF derived from the high-redshift, X-ray-selected 160 Square Degree ROSAT Cluster Survey (160SD) by Mullis et al. (2004) in a $\Lambda$-dominated universe, assuming the local XLF determined from the REFLEX survey (Böhringer et al. 2002). For consistency with the $160 \mathrm{SD}$, where $L_{\mathrm{X}}$ is calculated in the $0.5-2.0 \mathrm{keV}$ band, we derive the $P_{1.4}-L_{\mathrm{X}}$ correlation for mini-halos by estimating $L_{X}$ in the same energy band. For each cluster in our minihalo sample, we take the X-ray luminosity from the meta-catalogue of Piffaretti et al. (2011), which is given in the 0.1-2.4 keV energy band inside the radius $R_{500}$, and convert it to the $0.5-2.0 \mathrm{keV}$ energy band assuming an Xspec mekal plasma model at the observed cluster temperature (taken from Cavagnolo et al. 2009), redshift, and assuming a metallicity of 0.3. We derive a correlation in the form $\log P_{1.4}=2.03( \pm 0.20) \log L_{\mathrm{X}}-1.65( \pm 0.21)$, where $P_{1.4}$ is in units of $10^{24} \mathrm{~W} \mathrm{~Hz}^{-1}$ and $L_{\mathrm{X}}$ is in units of $10^{44} \mathrm{erg} \mathrm{s}^{-1}$. Hence in Eq. 3.2 we can substitute

$$
\frac{d L_{\mathrm{X}}}{d P_{1.4}}=\frac{L_{\mathrm{X}}}{P_{1.4}} \frac{d \log L_{\mathrm{X}}}{d \log P_{1.4}}=\frac{1}{2.03} \frac{L_{\mathrm{X}}}{P_{1.4}}
$$

By further assuming $\mathrm{f}_{\mathrm{SCC}} \sim 0.40$ (Hudson et al. 2010), we estimate the number counts of radio mini-halos as a function of redshift from the All Sky surveys $(3 \pi)$ with SKA1, early SKA1 and SKA2 (see Fig. 3, right panel); we predict that they will be able to detect up to $\sim 620, \sim 330$ and $\sim 1900$ mini-halos, respectively, out to redshift $z \sim 0.6$. If the cluster XLF is taken as known, a measure of the error on such estimates can be obtained by considering the combined maximum variation of the $P_{1.4}-L_{X}$ correlation parameters. This leads to uncertainties of the order of $\sim 30 \%$, $\sim 40 \%$ and $\sim 50 \%$ at $\mathrm{z}=0.1,0.3$ and 0.6 , respectively.

\section{BCG radio properties and interaction with the ICM as a function of cosmic epoch}

The radio-mode feedback is expected to be efficient in any cool core, in order to prevent complete cooling and provide a smooth temperature profile in the ICM as observed (McNamara et al. 2006; Rafferty et al. 2008). The presence of a radio galaxy in the BCG of a cluster is predicted to be generally associated to cavities in the ICM, as a result of the interaction between the population of relativistic electrons and the thermal electrons of the diffuse, X-ray emitting plasma. The study of cavities in the ICM inflated by relativistic electrons has been the subject of several studies aimed at understanding the energetics of the feedback processes, and the mechanism by which the mechanical energy is transferred to the ICM. The energetics can be estimated thanks to the enthalpy (4PV for relativistic plasma) of the bubbles (Bîrzan et al. 2004; Bîrzan et al. 2008), while the heating mechanism is still strongly debated. This mechanism is important also because it is thought to be responsible for the heating of the gas at galactic scales, efficiently quenching the star formation processes and therefore setting the stellar mass scales and colors for massive galaxies (Croton et al. 2006). In addition, strong radio sources can be the beacon of massive protoclusters at redshift 2 and larger (see, e.g., Miley et al. 2006). Therefore, radio selection of clusters and protoclusters may be an efficient method to find the progenitor of massive clusters at $z>2$, where the red sequence of the cluster galaxies may not be fully established, and the ICM is not shining in the X-ray band yet, indicating an incomplete virialization (Chiaberge et al. 2010). 


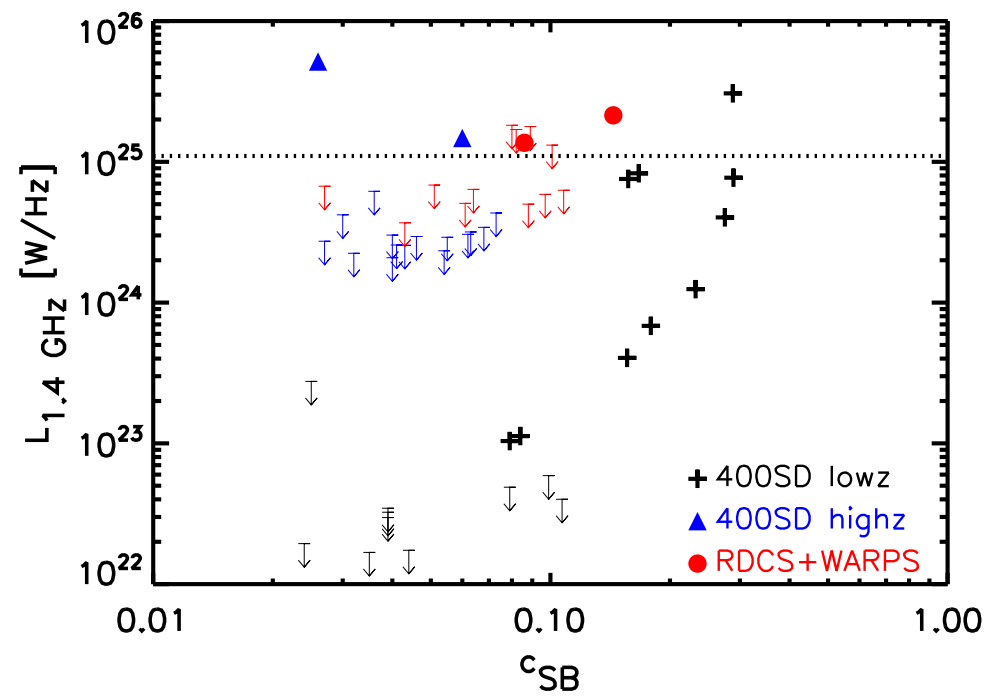

Figure 4: Relation between the radio luminosity of sources in close proximity to the cluster cores, $L_{1.4}$ $\mathrm{GHz}$, and the surface brightness concentration, $C_{S B}$. Arrows refer to upper limits of non-detections. The dotted line at $1.1 \times 10^{25} \mathrm{~W} \mathrm{~Hz}^{-1}$ marks the luminosity limit at $z=1$ for NVSS sources, corresponding to the flux limit of $2.5 \mathrm{mJy}$ (from Santos et al. 2010).

Tracing the feedback in galaxy clusters in the radio band consists of detecting the presence of radio AGN in the central galaxy, measuring its total power and resolving the radio emission in the radio lobes filling the cavities in the ICM. These studies have been very successful for local targets, where deep radio and X-ray data are both available, but they become increasingly difficult at higher redshifts. Recently, a few studies focused on high-redshift clusters and on the evolution of their properties with cosmic time. In particular, it has been realized that the evolution of the cool-core phenomenon is mirrored by the evolution of the feedback process itself. A simple correlation of the radio power and the cool-core strength (quantified through the concentration parameter $C_{S B}$ ) has been shown for low and medium redshift clusters (see Figure 4 right panel, Santos et al. 2010). It is observed that the luminosity of radio galaxies in the center of cool-core cluster have radio powers ranging from $\sim 10^{23} \mathrm{~W} \mathrm{~Hz}^{-1}$ to $10^{25} \mathrm{~W} \mathrm{~Hz}^{-1}$. Ongoing JVLA programs (PI P. Tozzi) are being carried out to target the majority of the high redshift clusters with X-ray data known to date, to extend this relation up to $z \sim 1$, and to perform a systematic investigations of the radiomode feedback in a representative sample of massive clusters in the CLASH sample (Postman et al. 2012). A comprehensive study of the radio properties of BCGs in X-ray selected galaxy clusters is in preparation (Hogan et al., submitted), in which multi-frequency radio observations allow one to investigate how the BCG properties vary with cluster environmental factors.

From a simple visual inspection of Figure 4, we conclude that we need to detect every radio galaxy at least down to $10^{23} \mathrm{~W} \mathrm{~Hz}^{-1}$ (at $1.4 \mathrm{GHz}$ ) in order to explore the same dynamical range 
at high redshift. In the left panel of Figure 5 we show the radio power in unresolved sources corresponding to detection limits of 20,10 and $1 \mu \mathrm{Jy}$ from top to bottom. Assuming that typically a detection requires a signal to noise ratio $S / N \sim 5$, these values correspond to $4 \mu \mathrm{Jy}, 2 \mu \mathrm{Jy}$ and $0.2 \mu \mathrm{Jy}$ per beam rms, which will be achieved by SKA1 (50\% sensitivity), SKA1 All Sky and SKA1 Deep Tier, respectively. With early SKA1 (50\% sensitivity) it will be possible to detect all the feedback process involving radio galaxies with power larger than $10^{23} \mathrm{~W} \mathrm{~Hz}^{-1}$ up to $z \leq 1$. We find that sources above $10^{23} \mathrm{~W} \mathrm{~Hz}^{-1}$ can be detected up to redshift $z \sim 1.7$ (the largest redshift where virialized galaxy clusters are currently found) and beyond with a sensitivity of $10 \mu \mathrm{Jy}$ per beam, which is easily achieved by SKA1-MID on the entire sky $(3 \pi)$ with a subarcsec resolution. At this sensitivity, confusion is avoided for a beam FWHM smaller than $4 \operatorname{arcsec}$ (see Prandoni $\&$ Seymour 2015). A ten time lower detection threshold, corresponding to an rms of $0.2 \mu \mathrm{Jy}$ per beam (blue line in Figure 5, left panel) is reached in the Deep Tier (2000 hrs) reference surveys (over about 10-30 $\mathrm{deg}^{2}$ ) with SKA1-MID. This sensitivity is obtained at an angular resolution below 1 arcsec, thus easily allowing a complete census of the radio properties of the BCG at any redshift down to a total power of $10^{22} \mathrm{~W} \mathrm{~Hz}^{-1}$ at $1.4 \mathrm{GHz}$. In this case, even in the presence of a strong negative evolution with redshift, all the BCG in massive clusters with cool core and feedback activity will be detected and characterized. Considering that on the entire sky there are potentially $4 \times 10^{4}$ massive clusters at $z>0.5$ which are within the reach of Athena or any X-ray survey mission with an average resolution of $\leq 10 \operatorname{arcsec}$ (like the Wide Field X-ray Telescope ${ }^{5}$ ), the number of galaxies with radio activity in cool cores at $z>0.5$ can be as high as $\sim 2 \times 10^{4}$, assuming little or no evolution in the population of cool core clusters, or few thousands assuming a strong evolution by a factor of 10 . At present, there is large uncertainty on the evolution of cool cores (Santos et al. 2010; McDonald et al. 2013), and only a strong synergy of SKA1 with deep and wide X-ray data will answer to this question. Clearly SKA, when completed, will allow one to investigate the presence of radio galaxies also in groups at high redshift, a completely new window for which it is impossible to make predictions given the present knowledge of the feedback processes. SKA therefore offers a unique opportunity to constrain the duty cycle of radio galaxies in groups and cluster of galaxies across the cosmic epochs. Another relevant aspect to consider is that radio BCGs often show variabiliy and a high complexity in their radio spectra (Hogan et al., submitted), with $\sim 80 \%$ of them showing a flat-spectrum/self-absorbed core. These flatter/active components will often be distinguishable only at frequencies larger than $5 \mathrm{GHz}$, making it desirable to have coverage at these frequencies.

A further major breakthrough in the investigation of radio-mode AGN feedback at high redshift is the detection and characterization of the radio lobes produced by the relativistic electrons which are responsible for carving the cavities in the ICM clearly observed in X-ray local clusters. If we use as a reference the bubble size in the two objects shown in Figure 1, we can directly compute the typical angular size of the bubbles as a function of the redshift. For the group HCG $62(z=0.0137)$ the average size for a single bubble, assuming a spherical shape, is $5 \mathrm{kpc}$, while for the mediummass cluster RBS $797(z=0.35)$ is $12 \mathrm{kpc}$. If we focus on targets at $z>1$ the angular size turns out to be about 0.6 and 1.5 arcsec, respectively, with a weak dependence on the redshift. For these objects we measure a total radio power at $1.4 \mathrm{GHz}$ of $2.0 \times 10^{21} \mathrm{~W} \mathrm{~Hz}^{-1}$ and $10^{24} \mathrm{~W} \mathrm{~Hz}^{-1}$ for

\footnotetext{
${ }^{5}$ see www.wfxt.eu
} 

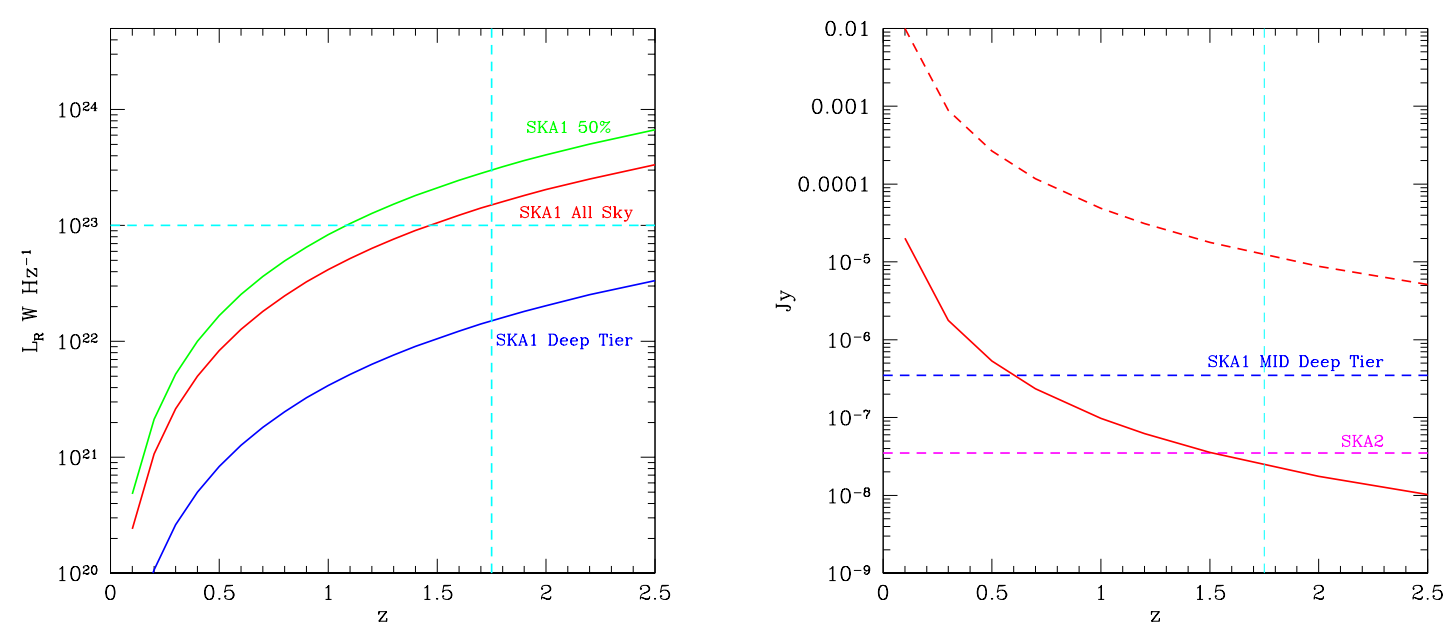

Figure 5: Left panel: Radio power at $1.4 \mathrm{GHz}$ detectable with a sensitivity of 1-10-20 $\mu J y$ (defined as $5 \times$ rms noise) are shown in blue, red, and green lines, respectively, as a function of redshift. Green corresponds to early SKA, red to SKA1 All Sky, and blue to SKA1 Deep Tier. The horizontal dashed line shows the minimum radio luminosity currently measured in a radio galaxy hosting a cool core in local clusters. Right panel: the dashed red line shows the flux density per beam with a resolution of 0.75 arcsec for a bubble with radio power of $10^{24} \mathrm{~W} \mathrm{~Hz}^{-1}$ as observed in the cluster RBS 797. The dashed horizontal blue line marks the sensitivity of Deep Tier (2000 hrs) reference surveys with SKA1-MID at the same angular resolution. The solid red line shows the flux density per beam with a resolution of 0.3 arcsec for a bubble with radio power of $2 \times 10^{21} \mathrm{~W} \mathrm{~Hz}^{-1}$ as observed in the group HCG 62 . The magenta dashed line shows the sensitivity reacheble with SKA2. In both panels the vertical dashed lines mark the highest redshift where virialized, $\mathrm{X}$-ray emitting clusters are currently detected.

a single cavity in HCG 62 and RBS 797, respectively. We conservatively assume that a beam FWHM of half the size of the bubble is sufficient to resolve it. Therefore, we compute the radio flux density at $1.4 \mathrm{GHz}$ from a single cavity as a function of redshift, assuming a spectral index $\alpha \sim 1$, and a beam FWHM of 0.3 arcsec and 0.75 arcsec for the reference cases of HCG 62 and RBS 797, respectively. We show in Figure 5 (right panel) that the signal from a single cavity in medium and large clusters is always well above the sensitivity of Deep Tier (2000 hrs) reference surveys with SKA1-MID, which is computed as $5 \times 70$ nJy per beam rms (see Prandoni \& Seymour 2015). At this resolution, we are also well above the confusion level. Therefore the detection and characterization of typical cavities in medium or large mass cluster is within the reach of SKA1MID surveys at any relevant redshift.

On the other hand, to detect a single cavity in a small group a beam FWHM of 0.3 arcsec is required, and given the much lower radio power of $\sim 2.0 \times 10^{21} \mathrm{~W} \mathrm{~Hz}^{-1}$, the expected radio flux density in the beam rapidly falls below the sensitivity limit of SKA1-MID (see red lines in Figure 5, right panel). According to the current specifics, SKA1-MID allows detection and characterization of radio bubbles only up to $z \sim 0.5$. If a $\sim 10$ times better sensitivity can be reached with SKA2 at the resolution of $0.3 \mathrm{arcsec}$, the investigation of the radio-mode feedback becomes feasible also in groups up to $z \sim 1.3$. We also note that the fraction of BCGs that show clear radio lobes in local X-ray selected clusters is about 10\% (Hogan et al., submitted). This leaves room for several 
thousands of clusters in the entire sky potentially hosting bubbles and cavities, but it may also indicate that most of the activity we actually observe around radio galaxies is either the aftermath of lobe creation and dissipation, or, more likely, low power outbursts that are confined within a few hundreds pc of the core. In this case at high redshift the full resolution SKA images will likely appear unresolved or amorphous. Nevertheless, the subarcsec angular resolution provided by the long baselines are essential to investigate the feedback process in details in relatively low and medium redshift clusters.

To summarize, our preliminary feasibility study shows that the radio-mode feedback will be unveiled practically at any level in clusters up to $z \sim 1.7$, the maximum redshift where virialized clusters have been detected in the X-ray band so far. The same study will be possible also in high-redshift groups if SKA2 will improve the sensitivity by an order of magnitude at the same angular resolution. When SKA will be fully operational, the knowledge of distant galaxy clusters will be largely but unpredictably changed. A large number of optically and IR selected clusters will be available thanks to the forthcoming surveys of the Euclid ${ }^{6}$ satellite and of the LSST project ${ }^{7}$. Thanks to the ICM X-ray characterization of the cluster cores achievable with the Athena mission, these can be directly combined with the radio data to search for cavities. However, an efficient synergy with SKA will depend on the on-axis angular resolution of the X-ray telescope, which should be of the order of 1 arcsec. In the field of high-z clusters, as in many others, the advent of SKA will provide access to incredibly deep and high quality data, which are hardly matched by any planned of foreseen facility in other wavebands.

\section{References}

Akritas, M. G. \& Bershady, M. A. 1996, ApJ, 470, 706

Ascasibar, Y. \& Markevitch, M. 2006, ApJ, 650, 102

Best, P. N. et al.. 2007, MNRAS, 379, 894

Bharadwaj, V. et al.. 2014, ArXiv e-prints

Bîrzan, L. et al.. 2008, ApJ, 686, 859

Bîrzan, L. et al.. 2004, ApJ, 607, 800

Böhringer, H. et al.. 2014, ArXiv e-prints

-. 2002, ApJ, 566, 93

Bonafede, A. et al.. 2014, MNRAS, 444, L44

Brunetti, G. et al.. 2009, A\&A, 507, 661

Brunetti, G. \& Jones, T. W. 2014, International Journal of Modern Physics D, 23, 30007

Burns, J. O. 1990, Astronomical Journal, 99, 14

Cassano, R. et al.. 2015, "Cluster Radio Halos at the crossroads between astrophysics and cosmology in the SKA era", in proceedings of "Advancing Astrophysics with the Square Kilometre Array", PoS(AASKA14)073

-. 2012, A\&A, 548, A100

-. 2008, A\&A, 486, L31

Cavagnolo, K. W. et al.. 2009, ApJ Supplement Series, 182, 12

\footnotetext{
${ }^{6} \mathrm{http} / / / \mathrm{sci} . e s a . i n t /$ euclid/

${ }^{7}$ http://www.lsst.org/lsst/
} 
Chiaberge, M. et al.. 2010, ApJ, 710, L107

Croton, D. J. et al.. 2006, MNRAS, 365, 11

Doria, A. et al.. 2012, ApJ, 753, 47

Dunn, R. J. H. \& Fabian, A. C. 2006, MNRAS, 373, 959

Fabian, A. C. 1994, Annual Rev. of Astronomy \& Astrophysics, 32, 277

—. 2012, Annual Rev. of Astronomy \& Astrophysics, 50, 455

Fanaroff, B. L. \& Riley, J. M. 1974, MNRAS, 167, 31P

Farnsworth, D. et al.. 2013, ApJ, 779, 189

Feretti, L. et al.. 2012, Astronomy \& Astrophysics Rev., 20, 54

Ferrari, C. et al.. 2015, "Non-thermal emission from galaxy clusters: feasibility study with SKA", in proceedings of "Advancing Astrophysics with the Square Kilometre Array", PoS(AASKA14)075

Fujita, Y. et al.. 2004, Journal of Korean Astronomical Society, 37, 571

Giacintucci, S. et al.. 2014a, ArXiv e-prints

-. 2014b, ApJ, 781, 9

Gitti, M. et al.. 2012, Advances in Astronomy, 2012

-. 2004, A\&A, 417, 1

-. 2002, A\&A, 386, 456

-. 2007, A\&A, 470, L25

-. 2013, A\&A, 557, L14

-. 2010, ApJ, 714, 758

Govoni, F. et al.. 2015, "Cluster magnetic fields through the study of polarized radio halos in the SKA era", in proceedings of "Advancing Astrophysics with the Square Kilometre Array", PoS(AASKA14) 105

Hlavacek-Larrondo, J. et al.. 2012, MNRAS, 421, 1360

Hudson, D. S. et al.. 2010, A\&A, 513, A37

Kale, R. et al.. 2013, A\&A, 557, A99

Markevitch, M. \& Vikhlinin, A. 2007, Physics Reports, 443, 1

Mazzotta, P. \& Giacintucci, S. 2008, ApJ, 675, L9

McDonald, M. et al.. 2013, ApJ, 774, 23

McNamara, B. R. \& Nulsen, P. E. J. 2012, New Journal of Physics, 14, 055023

McNamara, B. R. et al.. 2006, ApJ, 648, 164

Miley, G. K. et al.. 2006, ApJ, 650, L29

Mittal, R. et al.. 2009, A\&A, 501, 835

Mullis, C. R. et al.. 2004, ApJ, 607, 175

Murgia, M. et al.. 2009, A\&A, 499, 679

Panagoulia, E. K. et al.. 2014, MNRAS, 438, 2341

Peterson, J. R. \& Fabian, A. C. 2006, Physics Reports, 427, 1

Piffaretti, R. et al.. 2011, A\&A, 534, A109

Postman, M. et al.. 2012, ApJ Supplement Series, 199, 25

Prandoni, I. \& Seymour, N. 2015, "Revealing the Physics and Evolution of Galaxies and Galaxy Clusters with SKA Continuum Surveys", in proceedings of "Advancing Astrophysics with the Square Kilometre Array", PoS(AASKA14)067 
Rafferty, D. A. et al.. 2008, ApJ, 687, 899

Santos, J. S. et al.. 2010, A\&A, 521, A64

-. 2012, A\&A, 539, A105

Sun, M. 2009, ApJ, 704, 1586

van Weeren, R. J. et al.. 2014, ApJ, 786, L17

Zhuravleva, I. et al.. 2014, ArXiv e-prints

ZuHone, J. A. et al.. 2013, ApJ, 762, 78

—. 2010, ApJ, 717, 908 